\title{
Multilinguales
}

\section{Stylistique d'un texte de Victor Hugo en didactique du FLE}

Stylistics of a Text of Victor Hugo in Teaching French as a Foreign Language (F.F.L)

\section{Ayih Ayitey}

\section{OpenEdition}

\section{Journals}

Édition électronique

URL : http://journals.openedition.org/multilinguales/1175

DOI : 10.4000/multilinguales. 1175

ISSN : 2335-1853

\section{Éditeur}

Université Abderrahmane Mira - Bejaia

Édition imprimée

Date de publication : 1 décembre 2014

Pagination : $2-29$

ISSN : 2335-1535

\section{Référence électronique}

Ayih Ayitey, «Stylistique d'un texte de Victor Hugo en didactique du FLE », Multilinguales [En ligne], 4 | 2014, mis en ligne le 01 décembre 2014, consulté le 17 septembre 2019. URL : http://

journals.openedition.org/multilinguales/1175; DOI : 10.4000/multilinguales. 1175

Ce document a été généré automatiquement le 17 septembre 2019.

\section{c) (i) $९($}

Multilinguales est mise à disposition selon les termes de la Licence Creative Commons Attribution -

Pas d'Utilisation Commerciale - Pas de Modification 4.0 International 


\title{
Stylistique d'un texte de Victor Hugo en didactique du FLE
}

\author{
Stylistics of a Text of Victor Hugo in Teaching French as a Foreign Language \\ (F.F.L)
}

Ayih Ayitey

Dans cet article, nous proposons de construire une " unité didactique ${ }^{1}$ à partir d'un extrait des Misérables de Victor Hugo prisé par les pédagogues, à savoir l'arrivée de Jean Valjean à Digne (Annexe $n^{\circ} 1$ ). Notre méthode, dont la finalité est la double initiation à la langue et au texte littéraire des apprenants d'un niveau avancé ${ }^{2}$ en français langue étrangère, est organisée en six étapes, celle des préliminaires méthodologiques non incluse.

La première est une initiation audio-orale aux structures auxquelles l'apprenant sera confronté dans le texte littéraire. Celles-ci sont scindées, graduées et présentées sous forme de quatre dialogues, articulés autour d'un même thème, lisible dans le titre global, « à propos d'un voyageur », et numérotés de 1 à 4 (Annexe $n^{\circ} 2$ ).

Après cette étape préparatoire, s'en suit une seconde dont l'objectif est la compréhension globale du texte, à travers une série de questions-réponses entre les apprenants et l'enseignant (Annexe $n^{\circ} 3$ ).

La troisième étape consiste en une focalisation sur le thème dominant, à savoir le portrait du voyageur, appréhendé à travers le lexique de la description (Annexe ${ }^{\circ} 4$ ).

La quatrième étape vise la compétence grammaticale sur le mode inductif : la catégorie de l'adjectif est implicitement appréhendée selon sa position par rapport au substantif auquel il se rapporte : épithète, attribut, apposé (Annexe $n^{\circ}$ 5).

A la cinquième étape, l'apprenant est initié au style littéraire, en comparant la syntaxe des dialogues à celle du texte, et en réemployant, dans une production personnelle, les ressources de la description en situation de simulation (Annexe $\mathrm{n}^{\circ} 6$ ).

La sixième étape élargit, à partir du texte, le cercle de l'enseignement-apprentissage au contexte référentiel et à la culture du pays de langue. 
2 Ainsi, à partir de dialogues fabriqués ${ }^{3}$ en fonction des éléments ciblés dans un texte littéraire choisi au préalable, nous entrons progressivement dans le texte pour, finalement, en sortir et atteindre la sphère culturelle du référent.

\section{Préliminaires methodologiques}

Il est vrai que les textes littéraires n'ont eu une place notable dans l'apprentissage de la langue que dans la première méthodologie, la méthodologie traditionnelle, dite méthodologie de grammaire-traduction. Celle-ci, en effet, reposait essentiellement, pour ne pas dire exclusivement, sur la traduction des textes littéraires en langues étrangères, par le biais d'un exercice privilégié, le «thème ». Cependant, ces textes n'étaient pas étudiés du point de vue de leur spécificité littéraire, mais comme des morceaux choisis pour servir de modèles formels de la langue soutenue, à mémoriser et à imiter.

Mais, vers la fin du $19^{\text {ième }}$ siècle, la tendance s'est orientée vers la langue de communication au détriment de la langue littéraire. Ainsi, la méthode directe, qui s'est imposée dans l'enseignement des langues étrangères au début du $20^{\text {ième }}$ siècle, va donner la priorité à la langue orale, comme moyen de communication. Celle-ci précédait l'écrit conçu comme de l'« oral scripturé » (Bailly, 1997 :53), parce qu'il servait à fixer ce qui a été appréhendé à l'oral.

Des années 20 aux années 60, la méthodologie active, moins intransigeante que la précédente, va réintroduire le texte littéraire. Cependant, dans les années 60 , les méthodologies audio-orale (MAO) et structuro-globale et audio-visuelle (SGAV) vont de nouveau redonner la priorité à la langue orale et aux documents non authentiques (textes fabriqués ou transformés à des fins didactiques).

Il faut attendre les années soixante-dix et l'approche communicative pour la réintroduction des textes littéraires en tant que documents authentiques mais appréhendés d'un autre point de vue que celui de la méthodologie traditionnelle. En effet, selon J.-P. Cuq et I. Gruca (2005: 158) :

Avec l'approche communicative, la littérature est d'une certaine manière réhabilitée par

l'introduction des textes littéraires parmi les supports d'apprentissage. (...) Elle permet (...) d'appréhender le texte littéraire dans ses spécificités langagières, typologiques, génériques et thématiques.

Ainsi, dans cette approche, la langue du texte littéraire est considérée dans sa littérarité, au niveau sémantique, syntaxique, morphosyntaxique, avec une ouverture sur le contexte au niveau culturel, comme le souligne G. Mounin (1976:53) :

La littérature reste considérée comme la seule et toujours la meilleure ethnologie de la culture d'un pays donné, au sens propre du mot ethnographie: presque toutes les images et les idées les plus tenaces et les plus concrètes que nous avons sur les Anglais, les Russes ou les Grecs [...] sont venues [...] des [...] œuvres littéraires.

Mais, d'après de nombreux didacticiens dont C. Puren (2006), on ne peut parler, dans la perspective de l'approche communicative, d'une présence pleine et entière du texte littéraire parce qu'il est au centre d'une situation de communication artificielle, fictive, puisque simulée en classe en analogie avec des situations de communication réelles en société.

L'accentuation de la tendance de l'apprentissage d'une langue pragmatique, dictée par le contexte socioéconomique de ces dernières décennies, explique en grande partie que la méthodologie actionnelle prônée par le Cadre Européen Commun de Référence pour 
les Langues (CECR), dans les années quatre-vingt-dix et réajustée au début des années 2000, ait renoué avec la méthodologie active. D'après Christian Puren (2006) :

(...) La "perspective actionnelle" ébauchée dans le Cadre Européen commun de Référence pour les langues (CECR) de 2001 amorce une sortie de l'approche communicative et annonce une nouvelle conception d'ensemble de l'enseignement-apprentissage des langues qui permettent de " revisiter » historiquement la méthode active et de repenser la didactique du texte littéraire en classe de langue".

Ainsi, l'approche actionnelle se rapproche de la méthodologie active tout en se distinguant de l'approche communicative.

Certains, comme C.Puren (2006), vont jusqu'à parler de rupture entre les deux approches :

La perspective actionnelle et la méthode active (...) ont en commun de se fonder sur l'agir en général de l'apprenant - et sur l'agir collectif -, et non, comme l'approche communicative, sur une seule forme très particulière d'activité, celle de la communication langagière interindividuelle. (...) Dans l'approche communicative, on fait communiquer entre eux les élèves en classe pour qu'ils apprennent à communiquer en société.

Ainsi, l'approche actionnelle récuse ce type de simulation qui repose sur un «fairesemblant ", en tant que fin en soi ${ }^{5}$, incompatible avec sa conception de l'apprenant : un acteur social qui utilise la langue pour accomplir des tâches langagières et autres, insérées dans un projet global, dans un contexte social donné, dans un but déterminé ${ }^{\text {. }}$ Dans ce cadre, l'apprentissage conçoit l' "agir scolaire » dans sa dimension d'usage social (Conseil de l'Europe, 1976, 2001).

Force est de constater, cependant, que les méthodologies ne s'opposent jamais radicalement parce que chacune d'elle, succédant à l'autre ou concomitante par rapport à l'autre, se définit par rapport à elle ou vient la corriger, sans vraiment la supplanter sur le terrain. C'est ce qui expliquerait la tendance actuelle vers l'éclectisme au niveau pragmatique ${ }^{7}$.

5 En ce qui nous concerne, la méthode que nous proposons pour l'élaboration de notre proposition d'unité didactique croise plusieurs méthodologies :

- la méthode directe pour, notamment, l'approche par le lexique, l'enseignement implicite de la grammaire et les exercices de questions-réponses sous le contrôle de l'enseignant,

- la méthode active pour le choix de la situation pédagogique proche de la réalité, le recours à la simulation dicté par le souci de la motivation de l'apprenant,

- les méthodologies audio-orale et audio-visuelle pour le recours aux dialogues fabriqués par l'enseignant (texte oral «scripturé») en remplacement du texte écrit (même s'ils ne font que précéder le texte écrit dont ils sont inspirés),

- l'approche communicative pour l'échange interactif entre élèves, en classe, par le biais de "dialogues de base ", le recours à la fois à la simulation et aux documents authentiques (et donc aux textes littéraires), l'importance accordée au sens et à la compréhension sur la forme,

- l'approche actionnelle pour la conception de l'unité didactique sous forme de projet constitué de tâches orales et écrites.

Pour élaborer notre unité didactique, nous avons tenu compte du niveau (avancé) des apprenants et des objectifs pédagogiques à atteindre: faire acquérir le lexique du portrait, initier implicitement à la catégorie grammaticale de l'adjectif, sensibiliser au style littéraire par comparaison avec le style de la langue usuelle, ouvrir l'enseignement sur des réalités extratextuelles (culturelles, historiques, sociologiques, etc.) du pays de la langue. 
Le choix du texte littéraire adéquat a été fait en fonction de ces paramètres. Nous avons retenu un extrait des Misérables de Victor Hugo parce que ce roman, un classique de stature internationale, est une vaste fresque historique, sociale et humaine, dont les thèmes principaux (la misère, l'injustice, etc.) sont encore d'actualité et suscitent la compassion et l'empathie pour les personnages principaux, dont Jean Valjean, à même de motiver l'apprenant.

Le passage, qui décrit l'entrée à Digne d'un voyageur inconnu des habitants et qui n'est autre que Jean Valjean, est à ce point exemplaire qu'il a retenu l'attention de bon nombre de pédagogues.

\section{II. Étape préliminaire : dialogues audiovisuels de base}

6 Dans le cadre de notre article, l'approche proposée permet de lier l'étude de la langue et l'étude (stylistique) de la littérature en vue de favoriser l'acquisition de compétences orales, écrites et culturelles.

Il s'agit donc d'examiner les éléments du discours par lesquels l'apprenant accède à la littérarité du texte tout en poursuivant son apprentissage de la langue. Convaincu que c'est en prenant conscience des grandes articulations du texte que l'apprenant peut progresser dans sa connaissance de la littérature, comme dans sa connaissance de la langue, nous avons porté une attention minutieuse à la manière d'aborder ce texte littéraire en FLE.

$7 \mathrm{Au}$ stade préliminaire de notre unité didactique, nous proposons que l'enseignant construise quatre dialogues, sous le titre " $A$ propos d'un voyageur », à partir du texte littéraire auquel ils empruntent les idées maîtresses et dans une certaine mesure le vocabulaire et certaines tournures syntaxiques simplifiées. Fidèles à son contenu, ces dialogues sont construits dans un style simple qui prépare les apprenants à pouvoir cerner le sens du texte littéraire. Cette phase préparatoire est conçue selon les principes de la maïeutique.

A cet effet, une version audio des dialogues écrits est réalisée grâce au logiciel TextALoud. L'enseignant fait écouter les dialogues, en classe, à tous les apprenants. Ensuite, les apprenants interagissent entre eux pour répéter ces dialogues dans le but d'en comprendre le sens. A cette phase de compréhension, doivent succéder les phases d'analyse et d'interprétation et de commentaire de ces dialogues, dans l'esprit de l'approche actionnelle. Ainsi, permettre aux apprenants de donner leur opinion contribue à leur conférer le statut d'« acteur social » qui les motiverait plus que celui de « simulateur » qu'ils ont dans l'approche communicative.

8 Le premier dialogue (annexe $n^{\circ} 1$ ) introduit les grandes articulations du texte proposé. Il s'agit d'un voyageur que les apprenants vont découvrir progressivement. En tenant compte de cette progression, le premier dialogue, court, sollicite des réponses à trois questions préliminaires au sujet du voyageur : qui ? Quand ? Où ?

Le second dialogue (annexe $n^{\circ} 1$ ) est plus long que le premier parce que l'intention est de progresser dans la connaissance de ce voyageur au sujet duquel le premier dialogue ne nous donne aucune information. Dans celui-ci, les apprenants vont commencer à le connaître parce qu'ils vont y trouver des éléments descriptifs et narratifs, inspirés de l'extrait hugolien, en particulier, son allure et ses faits et gestes. 
Ce dialogue prépare les apprenants plus directement que le précédent à la compréhension du texte littéraire, en justifiant l'état de « fatigue » du voyageur par des arguments contenus dans le texte.

10 Le troisième dialogue (annexe $\mathrm{n}^{\circ} 1$ ) donne plus de détails sur la description du voyageur amorcée au niveau du second. Les descriptions, à travers un vocabulaire spécifique, portent sur la façon dont il est habillé pour expliquer sa condition sociale et annoncer le thème de la misère sociale traité dans un autre point de l'unité didactique.

11 Le quatrième et dernier dialogue (annexe $n^{\circ} 1$ ) se situe dans le prolongement du troisième pour aborder quelques aspects du portrait physique du voyageur. Ce dialogue précise le point de vue ou la focalisation de la description du voyageur par son auteur.

Après avoir écouté, lu, analysé et commenté les dialogues, les apprenants sont sollicités pour les jouer sous forme de saynètes, comme préconisé dans la méthode active. Cette activité de dramatisation est destinée à contribuer à la motivation de l'apprenant, tout en consolidant sa compréhension des dialogues dans leur progression.

Ces dialogues sont conçus en tenant compte du niveau des apprenants, dont les besoins ont été déterminés en amont, et ensuite conceptualisés en activités, en prévision de la compétence linguistique à exploiter à travers le texte. En outre, destinés à faciliter la compréhension du texte littéraire, ils ont disposé les apprenants à sa "lecture de repérage » qui consiste, selon Christine Tagliante (2001: 126), à «rechercher des informations précises et ponctuelles $»^{8}$.

Il ne s'agit pas d'un simple enjeu de communication qui vise à sensibiliser par anticipation les apprenants à un certain lexique, mais d'une démarche pédagogique qui révèle une connaissance du niveau des apprenants. L'importance du texte littéraire en classe de FLE passe par ce détour inconditionnel dont l'objectif principal est de pouvoir l'introduire comme support quelles que soient les difficultés que présente ce type de texte au niveau de son style.

Après ces préliminaires, les apprenants peuvent procéder (avec l'aide de l'enseignant) à la recherche des repères stylistiques.

\section{La compréhension du texte}

Pour la compréhension du texte, le rôle de l'enseignant est de guider les apprenants. Il les aide à saisir le sens véhiculé par le support. Après la lecture du texte proposé, il doit procéder par une sorte de "maïeutique», procédé pédagogique qui consiste à " accoucher les esprits", suscite la réflexion intellectuelle par une série de questionsréponses. Cette méthode s'inscrit à la fois dans l'esprit de la méthodologie active, qui recommande les exercices conversationnels sous la direction de l'enseignant, et dans celui de l'approche communicative, qui permet la réflexion autour du texte littéraire dans une interaction qui rend la classe vivante. A ce sujet, J.-P. Cuq et I. Gruca (2005 : 415) affirment que

Cette démarche a l'avantage d'activer les connaissances diffuses que possède le lecteur dans sa culture d'origine, de favoriser une véritable interaction entre le texte et son lecteur et de susciter le plaisir du texte, qui selon Barthes, provient de la reconnaissance d'éléments connus et de la perception de ceux qui sont en effraction par rapport aux attentes du code général et littéraire.

Ainsi, l'enseignant va aborder, avec les apprenants l'exploitation du texte littéraire, à travers une série de questions auxquelles ils devront répondre. Certes, la démarche ne permet pas, à ce stade, une exploration du style de l'auteur, mais une familiarisation 
avec les caractéristiques générales d'un texte descriptif'. Ainsi, l'explication du texte hugolien va s'élaborer autour des foyers de connaissances construits par l'enseignant de façon progressive, dans une dynamique de "questions et réponses", avec des réponses puisées directement du texte.

Cette progression (annexe $n^{\circ} 3$ ) commence par ce qui a trait à l'arrivée du voyageur à Digne : quand est-il arrivé ? Comment est-il arrivé ? D'où venait-il ? Etait-il connu des habitants ?...

Elle s'étend, ensuite, aux faits et gestes de ce voyageur inconnu : A-t-il fait des haltes en arrivant à Digne ? Où s'est-il arrêté ? Pourquoi s'est-il arrêté ?...

Elle aborde, enfin, son allure physique: quel est son aspect? Quelle est sa corpulence ?...

13 À ce niveau, se forme déjà dans la conscience des apprenants, la représentation mentale de la situation narrative. Même si les circonstances de ce voyage et l'identité du voyageur ne sont pas précisées, l'essentiel du texte est appréhendé, à savoir le portrait du personnage entrant dans la ville et les différents lieux qu'il traverse et le moment où il le fait.

En outre, le lexique de la description du voyageur, qui dénote son statut social, est repéré par les apprenants. Cet aspect du texte est également important parce qu'il justifie l'une des fonctions pédagogiques de la littérature, selon Régis Malet (2010:56) : "la finalité de l'étude de la littérature est de construire la culture humaniste tout en développant la langue comme outil pour encourager la réflexion provoquée par la lecture ».

Ainsi, la démarche conduit, progressivement, à l'exploration du thème dominant du corpus.

\section{La focalisation sur le thème dominant}

L'investigation thématique permet d'aborder enfin le style de l'auteur. À cette étape, un travail de regroupement des lexèmes par champ lexico-sémantique permet de cerner le thème dominant du texte. Cette recherche lexicale est un moyen de dégager le vocabulaire du portrait, qui peut être ainsi mémorisé. Ainsi, par une série de questionsréponses, la classe parvient à identifier et à classer les mots et expressions qui renseignent sur le portrait physique, la tenue vestimentaire et la condition sociale $\mathrm{du}$ voyageur (voir annexe $n^{\circ} 4$ ).

Ce relevé, effectué en suivant la progression linéaire du texte, montre une focalisation exclusive sur l'aspect physique du voyageur en tant que révélateur de sa condition sociale, comme indiqué dans le tableau en annexe.

L'enseignant peut alors faire le lien entre l'indigence du voyageur et le thème dominant de tout le roman tel qu'affiché par son titre : Les Misérables.

L'apprenant se rend compte qu'à partir d'un thème (dominant) repéré dans un extrait, grâce au lexique, il lui est possible d'avoir une idée du sujet de l'œuvre tout entière qu'il peut alors vouloir lire avec plaisir. Susciter le plaisir de lire une œuvre littéraire chez les apprenants est, en effet, l'un des objectifs de l'enseignant de FLE.

Il est donc évident qu'en étudiant un texte littéraire en classe de FLE, la compréhension est une étape importante vers l'appropriation de la langue et l'ouverture vers la littérature et la culture. 


\section{L'appropriation implicite de la grammaire : les adjectifs}

Selon Georges Molinié (1980 : 20),

Le moyen le plus sûr pour tenter de caractériser un style - opération délicate - consiste à envisager systématiquement les divers constituants linguistiques de ce style, quels que soient la nomenclature et le regroupement adoptés. C'est ce qu'on va proposer ici, en essayant de sélectionner, parmi les traits typiques d'une fonction stylistique donnée, ceux qui paraissent davantage relever d'une manière particulière.

Parmi les traits typiques à relever dans ce texte de Victor Hugo, celui de la classe des adjectifs qualificatifs paraît statistiquement prédominant. A ce sujet, Molinié (1986 : 57) affirme que «la place de l'adjectif qualificatif par rapport au nom est un important facteur stylistique ».

Après le repérage de ce trait stylistique important, les apprenants formeront des groupes pour mener un travail d'investigation sur le sujet.

Cette recherche permet aux apprenants de relever, de classer les adjectifs qualificatifs selon leur fonction et de les présenter sous forme de tableau (annexe $n^{\circ} 5$ ).

Il est évident qu'un tel exercice concerne des apprenants qui ont déjà une notion concernant la catégorie grammaticale envisagée.

Précisons que les participes passés sont également relevés et classés avec les adjectifs qualificatifs. Les catégories d'adjectifs qualificatifs, épithète, attribut et la mise en apposition, sont illustrées à travers des modèles présentés en tête du tableau (voir annexe $\mathrm{n}^{\circ} 4$ ) pour que la grammaire reste implicite. L'abondant usage que fait Hugo des adjectifs qualificatifs, confirme les affirmations de Georges Molinié (1986: 43): «Si on revient aux voies classiques pour envisager le cas du nom, on devrait pouvoir dire que le caractérisant spécifique du nom est l'adjectif ".

Le style de Victor Hugo, dans cet extrait, le montre clairement à travers cet emploi massif des qualifiants et leur diversité : adjectifs qualificatifs, participes passés, adjectifs verbaux. Cet aspect grammatical est un signe de la richesse du texte hugolien qui offre de nombreux exemples d'épithètes et d'attributs qui permettent d'aborder des points annexes, comme la notion de participe (présent et passé) et celle des verbes d'état. Cependant, cet extrait n'offre pas d'exemple d'adjectif apposé dont il faudra souligner l'absence car, l'enseignant, à ce niveau, met l'accent sur certaines difficultés de l'emploi des adjectifs épithètes pour que les apprenants, en s'appropriant les nombreux adjectifs qualificatifs utilisés dans le texte, sachent en faire bon usage.

Ainsi, lorsque, par exemple, Victor Hugo écrit "la barbe longue», "les rares habitants ", " de moyenne taille ", " une vieille blouse grise ", l'apprenant, qui constate que la place de l'adjectif n'est pas fixe par rapport au nom, est amené à se poser des questions sur les incidences de ces différentes positions (antéposé, postposé, apposé) sur son sens.

Le sujet a déjà attiré l'attention des grammairiens :

Un énorme repas signifie-t-il la même chose qu'un repas énorme? (...) Bien avant Vaugelas, ce problème a intrigué les grammairiens : comment se fait-il que l'adjectif tantôt précède son substantif et tantôt lui succède? Voilà une particularité que la langue française a développée à la perfection: par l'emplacement de l'adjectif épithète on peut jouer sur des nuances sémantiques et stylistiques parfois très subtiles. Qui plus est, tantôt le même adjectif accepte les deux positions sans notable distinction de sens, tantôt l'antéposition et la postposition correspondent à deux valeurs nettement différentes. (Nølke, 1996 : 38) 
Il s'agit donc, pour l'enseignant, de prendre en considération cette préoccupation « des nuances sémantiques et stylistiques » de l'adjectif épithète en fonction de la place qu'il occupe par rapport au nom auquel il se rapporte, pour relever, notamment, des cas déterminés par convention d'usage :

- d'adjectifs antéposés, comme «homme pauvre » / «pauvre homme » (dialogue $n^{\circ} 3$ ), « de moyenne taille » (texte) en association avec les expressions lexicalisées « de petite taille » et « de grande taille », « les rares habitants qui... »,

- d'adjectifs postposés, comme dans « une casquette (...) rabattue » (texte), « la tête tondue et la barbe longue » (texte),

- d'adjectifs situés de part et d'autre du nom: «grosse toile jaune»/«vieille blouse grise »/« énorme bâton noueux » (texte).

Les facteurs, qui justifient la place de l'adjectif qualificatif épithète en français, dont certains relèvent d'une nécessité stylistique interne au texte, devront être identifiés et expliqués. Michel Arrivé, dans La Grammaire d'aujourd'hui: guide alphabétique de linguistique française (1986: 37-38), évoque, par exemple, la taille de l'adjectif, la structure du syntagme nominal, la structure du syntagme adjectival, ainsi que des facteurs sémantiques.

Mais, dans une classe de FLE où l'on vise une acquisition rapide des compétences linguistiques, il est difficile de rentrer dans des détails trop pointus concernant des règles sur d'interminables et imprécises exceptions grammaticales, d'autant que les règles auxquelles on devrait se fier pour le faire n'existent pas, comme le souligne Henning Nølke (1996 : 38) :

Peut-on espérer qu'une quelconque théorie linguistique puisse jamais rendre compte de telles subtilités? Quelles sont les règles? Car des règles, il y en a forcément. En effet, si consulter l'oreille est pertinent, comme le disait Vaugelas, cette consultation n'en est pas moins régie par des règles. Sinon, comment expliquer que toute oreille francophone donne à peu près la même réponse pour le même énoncé? Mais l'oreille non francophone? Elle a besoin de connaître ces règles. Or malgré de multiples essais judicieux, personne ne semble avoir réussi à découvrir, voire à expliquer, tous les mécanismes qui sous-tendent ce jeu complexe et subtil qui pose tant de problèmes aux non-francophones.

La citation n'a pas été tronquée volontairement, parce qu'elle met l'accent sur l'une des grandes difficultés à laquelle se heurtent les apprenants et les enseignants de FLE : de nombreux usages de la langue, comme dans le cas de l'adjectif épithète, sont régis par des conventions qui ne sont ni explicitées ni formalisées. Cette absence de règles grammaticales précises, dans certaines situations, met l'apprenant de FLE en difficulté dans sa compétence à utiliser correctement, dans d'autres circonstances que celles de la classe, les notions acquises.

Cependant, étudier les adjectifs qualificatifs, dans un texte littéraire, choisi précisément à cet effet, permet de compenser un tant soit peu cette défaillance, surtout lorsqu'il est retenu à bon escient. Ainsi, pour les adjectifs qualificatifs, le texte descriptif, comme ici, est particulièrement indiqué.

Ce choix est stratégique surtout lorsque, comme c'est le cas ici, l'initiation à la grammaire est implicite. Cette conception inductive de la grammaire, principe d'enseignement recommandé par plusieurs méthodologies (active, communicative, etc.), est ainsi définie dans Dictionnaire didactique des langues (R. Galisson et D. Coste, $1976: 254)$ :

La grammaire implicite vise à donner aux élèves la maitrise d'un fonctionnement grammatical, mais ne recommande l'explicitation d'aucune règle et élimine le métalangage, ne s'appuyant que sur une manipulation plus ou moins systématique d'énoncés et de formes. 
17 A ce stade, nous parvenons à la phase d'analyse du style de l'auteur, qui nécessite d'entrer de plain-pied dans le texte, suivie de celle du réemploi des structures apprises, donc de l'écriture.

\section{Analyse du style et écriture créatrice}

L'analyse du style littéraire peut se faire sur plusieurs plans. Ici, nous allons nous intéresser uniquement à la syntaxe. En comparant les phrases littéraires du texte d'Hugo avec les phrases simples des dialogues étudiés en amont (annexe $n^{\circ} 6$ ), et qui ont permis de faciliter la compréhension du texte, nous pensons sensibiliser l'apprenant au style littéraire. Selon Maurice-Jean Lefebvre (1969: 230) :

(...)Les enseignements de la stylistique et ceux de la thématique en montrant comment ils se complètent et comment leur réunion peut mettre en évidence l'essentiel qui est, non pas simplement de détecter, pour l'analyse, les structures signifiantes...mais de montrer ce que ces structures ont d'original, de proprement littéraire. [...] de se poser la question de savoir pourquoi un tel fonctionnement est la source de notre fascination.

À partir donc des structures des deux discours, l'enseignant doit mettre au jour la littérarité de la syntaxe hugolienne, tout en tentant d'expliciter en quoi elle est la "source de notre fascination ». Les apprenants découvrent, du point de vue stylistique, la construction phrastique du texte, une construction qui révèle la cohérence des énoncés.

A cette phase d'initiation au style littéraire, succède la phase de réemploi par le biais de productions écrites dans le but d'évaluer ce qui a été fixé oralement.

Le passage à l'écrit se fait de manière pratique. Le professeur prévoit pour la séance, le déguisement d'un homme pauvre, donc en " haillons ", comme dans le texte. L'idée est de suggérer sa condition sociale misérable par son allure. Pour cela, un apprenant se porte volontaire pour porter l'accoutrement prévu. Ce déguisement met la classe devant une situation quasi « réelle », dans laquelle le « misérable » n'est plus imaginé mentalement mais présent dans la réalité de la classe. Les apprenants peuvent interagir avec ce voyageur en lui posant des questions avant de le décrire par écrit.

Cette situation simulée motive l'apprenant et active les connaissances et les structures auxquelles l'apprenant a été initié au cours des étapes précédentes. Le passage à l'écrit est conçu comme le moyen de fixer ce que l'apprenant sait déjà employer oralement. Donc pour la compétence écrite, un exercice de composition libre sur ce voyageur déguisé est demandé.

Jusque-là nous avons exploité les compétences fondamentales que l'on retrouve dans les méthodologies active, communicative et actionnelle : comprendre (qui implique les compétences lire et écouter), parler et écrire.

Mais, cette étape ne marque pas la fin de notre séquence pédagogique parce qu'il nous faut introduire la dimension culturelle dans l'apprentissage du FLE.

\section{De la langue à la culture}

En nous référant à l'approche actionnelle, et surtout au CECR dans lequel elle s'inscrit, nous prenons en considération son principe de ne pas se contenter de faire de l'apprenant, un usager compétent dans la langue étrangère, mais de tenir compte de sa position d'« acteur social». 
Dans cet ordre d'idée, le texte du CECR précise que l'apprentissage en langue étrangère vise deux grands types de compétences: les "compétences générales individuelles " (les savoirs, les habiletés et savoir-faire, les savoir-être, les savoirs apprendre), et la "compétence à communiquer langagièrement». Cette dernière est composée de la "compétence linguistique», de la «compétence sociolinguistique» et de la « compétence pragmatique ». Il est donc indéniable que le texte littéraire en favorisant le perfectionnement linguistique de l'apprenant lui permet également d'acquérir des connaissances culturelles, à travers la compétence à communiquer.

Aussi incombe-t-il à l'enseignant de faire un choix pertinent du texte littéraire pouvant lui permettre d'opérer une ouverture sur la société et la culture. De ce point de vue, le texte hugolien l'est à plus d'un titre, comme le montre le montage de cette unité didactique. Pour l'exploitation de cette dernière étape, une phrase du texte, telle que " il faisait son entrée dans Digne par la même rue qui sept mois auparavant avait vu passer l'empereur Napoléon allant de Cannes à Paris ", offre de grandes possibilités d'exploitation. Par exemple, les patronymes, les toponymes, comme ceux contenus dans cet extrait, et les anthroponymes, sont des marqueurs historiques et culturels qui doivent être exploités en tant que tels, dans le cadre de la compétence à communiquer, et en particulier, la compétence pragmatique.

S'il faut admettre avec Zarate G. (1993 :37) que « comprendre une réalité étrangère, c'est expliciter les classements propres à chaque groupe et identifier les principes distinctifs d'un groupe par rapport à un autre ", alors Paris, Cannes, Digne et Napoléon sont des traits distinctifs de la société française; ils doivent donc, à ce titre, être explicités au niveau culturel, social et historique et géopolitique.

D'autres indicateurs culturels, dont l'œuvre de Victor Hugo regorge, seront également convoqués.

Quoiqu'assez complexe, tant au niveau de sa compréhension qu'au niveau de son analyse et de son interprétation, le texte littéraire en classe de FLE, a l'intérêt d'initier à la compétence linguistique, et aux connaissances culturelles, considérées comme indispensables à la prise de "conscience interculturelle» retenue par le CECR au niveau du «savoir » des "compétences générales » de l'apprenant. D’où l'importance stratégique du texte littéraire dans l'enseignement de la langue étrangère. Nous pensons l'avoir démontré dans l'esquisse de cette proposition d'unité didactique. L'originalité de la démarche proposée réside dans la mise en place d'une méthode qui s'inspire de différentes méthodologies d'enseignement du FLE pour une plus grande efficacité.

\section{BIBLIOGRAPHIE}

ARRIVE M., GADET F., GALMICHE M., La Grammaire d'aujourd'hui : guide alphabétique de linguistique française, Paris, Flammarion, 1986. 
BAILLY Danielle, Didactique de l'anglais, tome 1. Objectifs et contenus de l'enseignement, Paris, Nathan Pédagogie, 1997.

CONSEIL DE L'EUROPE, Un Cadre Européen Commun de Référence pour les Langues : apprendre, enseigner, évaluer, Paris, Didier, 2001.

CUQ J.-P. \& GRUCA I., Cours de didactique du français langue étrangère et seconde, Grenoble, Presses Universitaires de Grenoble, 2005.

GALISSON R., COSTE D., Dictionnaire didactique des langues, Paris, Hachette, 1976.

LEFEBVRE M.-J., « Critique imaginale et langage littéraire » in Revue des langues vivantes, t. XXXV, 1969.

MALET R., École, Médiations et réformes curriculaires : Perspectives internationales, Louvain-la-Neuve, De Boeck Supérieur, 2010.

MOLINIE G., Éléments de stylistique française, Presses Universitaires de France, 1986.

MOLINIE G., « Étude stylistique d'une page de Malraux : L'Espoir ( $\left.\mathrm{I}^{\circ}, \mathrm{II}-3\right)$ », in L'information grammaticale, $\mathrm{n}^{\circ} 4$, janvier 1980, p. 20-24.

MOUNIN G., Linguistique et traduction, Paris, Mardaga, 1976. NØLKE H., « Où placer l'adjectif épithète ? Focalisation et modularité ", in Langue française, $n^{\circ} 111,1996$, pp. 38-58.

PUREN C., La Didactique à la croisée des méthodes : essai sur l'éclectisme, Paris, Hatier/Didier, 1994.

TAGLIANTE C., La classe de langue, Paris, CLE International, 2001.

ZARATE G., Représentations de l'étranger et didactique des langues, Paris, Didier, 1993.

\section{ANNEXES}

\section{Annexe $\mathrm{n}^{\circ} 1$}

Dans les premiers jours du mois d'octobre 1815, une heure environ avant le coucher du soleil, un homme qui voyageait à pied entrait dans la petite ville de Digne. Les rares habitants qui se trouvaient en ce moment à leurs fenêtres ou sur le seuil de leurs maisons regardaient ce voyageur avec une sorte d'inquiétude. Il était difficile de rencontrer un passant d'un aspect plus misérable. C'était un homme de moyenne taille, trapu et robuste, dans la force de l'âge. Il pouvait avoir quarante-six ou quarante-huit ans. Une casquette à visière de cuir rabattue cachait en partie son visage brûlé par le soleil et le hâle et ruisselant de sueur. Sa chemise de grosse toile jaune, rattachée au col par une petite ancre d'argent, laissait voir sa poitrine velue; il avait une cravate tordue en corde, un pantalon de coutil bleu usé et râpé, blanc à un genou, troué à l'autre, une vieille blouse grise en haillons, rapiécée à l'un des coudes d'un morceau de drap vert cousu avec de la ficelle, sur le dos un sac de soldat fort plein, bien bouclé et tout neuf, à la main un énorme bâton noueux, les pieds sans bas dans des souliers ferrés, la tête tondue et la barbe longue.

La sueur, la chaleur, le voyage à pied, la poussière, ajoutaient je ne sais quoi de sordide à cet ensemble délabré. Les cheveux étaient ras, et pourtant hérissés; car ils commençaient à pousser un peu, et semblaient n'avoir pas été coupés depuis quelque temps.

Personne ne le connaissait. Ce n'était évidemment qu'un passant. D'où venait-il ? Du midi. Des bords de la mer peut-être. Car il faisait son entrée dans Digne par la même rue qui sept mois auparavant avait vu passer l'empereur Napoléon allant de Cannes à Paris. Cet homme avait dû 
marcher tout le jour. Il paraissait très fatigué. Des femmes de l'ancien bourg qui est au bas de la ville l'avaient vu s'arrêter sous les arbres du boulevard Gassendi et boire à la fontaine qui est à l'extrémité de la promenade. Il fallait qu'il eût bien soif, car des enfants qui le suivaient le virent encore s'arrêter et boire, deux cents pas plus loin, à la fontaine de la place du Marché. (Victor Hugo, Les Misérables, Livre II, chap. 1)

\section{Annexe $\mathrm{n}^{\circ} 2$}

\begin{tabular}{|c|c|}
\hline \multicolumn{2}{|l|}{ DIALOGUE $\mathbf{N}^{\circ} 1$} \\
\hline A- HIER, J'AI VU UN VOYAGEUR DANS CETTE RUE. & B - QUAND L'AS-TU VU? \\
\hline $\begin{array}{l}\text { A-JE L'AI VU AVANT LE COUCHER DU SOLEIL. IL AVAIT } \\
\text { UN SAC DE SOLDAT. }\end{array}$ & B- D'OÙ VENAIT-IL? \\
\hline $\begin{array}{l}\text { A-DU MIDI, JE PENSE. DES BORDS DE LA MER PEUT- } \\
\text { ÊTRE. }\end{array}$ & B- TU LE CONNAISSAIS ? \\
\hline A-NON, JE NE L'AI JAMAIS VU AUPARAVANT. & \\
\hline \multicolumn{2}{|l|}{ DIALOGUE $\mathrm{N}^{\circ} 2$} \\
\hline & B- DE QUOI AVAIT-IL L'AIR? \\
\hline $\begin{array}{l}\text { A-IL AVAIT L'AIR D'UN HOMME VISIBLEMENT } \\
\text { FATIGUÉ. }\end{array}$ & B- COMMENT LE SAIS-TU? \\
\hline $\begin{array}{l}\text { A-IL SUAIT DE PARTOUT ET AVAIT DE LA POUSSIÈRE } \\
\text { SUR SES HABITS. }\end{array}$ & $\begin{array}{l}\text { B- LA POUSSIÈRE...DONC IL A VOYAGÉ À } \\
\text { PIED? }\end{array}$ \\
\hline A- OUI, CAR IL AVAIT SOIF. & B- AVAIT-IL UNE GOURDE? \\
\hline $\begin{array}{l}\text { A-NON, JE NE PENSE PAS, CAR IL A BU À LA FONTAINE } \\
\text { QUI EST À L'EXTRÉMITÉ DE LA PROMENADE ET À LA } \\
\text { FONTAINE DE LA PLACE DU MARCHÉ. }\end{array}$ & B- IL VENAIT DE LOIN, PROBABLEMENT. \\
\hline \multicolumn{2}{|l|}{ A- BIEN-SÛR. CET HOMME DEVAIT VENIR DE LOIN. } \\
\hline \multicolumn{2}{|l|}{ DIALOGUE $N^{\circ} 3$} \\
\hline- & B- COMMENT ÉTAIT-IL HABILLÉ? \\
\hline A- IL AVAIT UN ASPECT MISÉRABLE. & $\begin{array}{l}\text { B- EST-CE PARCE QUE SES HABITS ÉTAIENT } \\
\text { POUSSIÉREUX QUE TU AS PENSÉ QU'IL } \\
\text { ÉTAIT PAUVRE? }\end{array}$ \\
\hline A- NON. IL AVAIT UN PANTALON USÉ ET TROUÉ. & $\begin{array}{l}\text { B- N’Y AVAIT-IL QUE SON PANTALON QUI } \\
\text { ÉTAIT USÉ ET TROUÉ? }\end{array}$ \\
\hline $\begin{array}{l}\text { A- NON, SA BLOUSE AUSSI ÉTAIT VIEILLE ET } \\
\text { RAPIÉCÉE. TOUT SON ASPECT ÉTAIT CELUI D'UN } \\
\text { HOMME PAUVRE. }\end{array}$ & $\begin{array}{l}\text { B- AH! PAUVRE HOMME! ÊTRE HABILLÉ DE } \\
\text { LA SORTE ET VOYAGER À PIED, QUELLE } \\
\text { MISÈRE! }\end{array}$ \\
\hline
\end{tabular}




\begin{tabular}{|l|l|}
\hline \multicolumn{2}{|l|}{ DIALOGUE N ${ }^{4} \mathbf{4}$} \\
\hline- & $\begin{array}{l}\text { B-OÙ ÉTAIS-TU QUAND LE VOYAGEUR EST } \\
\text { PASSÉ? }\end{array}$ \\
\hline A-J'ÉTAIS À MA FENÊTRE ET JE L'AI APERÇU DE LOIN. & \begin{tabular}{l} 
B-TU AS PABILLEMENT DE LOIN? DISTINGUER SON \\
\hline $\begin{array}{l}\text { A-NON, J'ÉTAIS DESCENDU AU SEUIL DE MA MAISON } \\
\text { QUAND IL S'ÉTAIT APPROCHÉ. }\end{array}$
\end{tabular} \\
\hline $\begin{array}{l}\text { A-BIEN SÛR, C'ÉTAIT UN HOMME QUU POUV AS PU LE VOIR DE PRÈS ? } \\
\text { QUARANTE-SIX OU QUARANTE-HUIT ANS. }\end{array}$ & B-QUELLE ÉTAIT SA CORPULENCE? \\
\hline A-IL ÉTAIT DE TAILLE MOYENNE, TRAPU ET ROBUSTE. & \\
\hline
\end{tabular}

\section{Annexe $n^{\circ} 3$}

\begin{tabular}{|c|c|}
\hline Questions & Réponses \\
\hline De qui parle l'auteur? & L'auteur parle d'un voyageur. \\
\hline Quel est le moyen de & «... un homme qui voyageait à pied \\
\hline $\begin{array}{l}\text { locomotion du personnage ? } \\
\text { Comment le sait-on? }\end{array}$ & entrait dans la petite ville de Digne» \\
\hline $\begin{array}{l}\text { Le voyageur connaissait-il les } \\
\text { habitants de la ville? }\end{array}$ & $\begin{array}{l}\text { Non, le voyageur ne connaissait pas les habitants de la ville et } \\
\text { personne ne l'a reconnu. }\end{array}$ \\
\hline $\begin{array}{l}\text { En quel mois et en quelle } \\
\text { année était-il entré dans la } \\
\text { ville de Digne? }\end{array}$ & $\begin{array}{l}\text { Il était entré dans la ville de Digne « dans les premiers jours du mois } \\
\text { d'octobre 1815, une heure environ avant le coucher du soleil». }\end{array}$ \\
\hline $\begin{array}{l}\text { Par quelle rue était-il entré } \\
\text { dans la ville? }\end{array}$ & $\begin{array}{l}\text { Il était entré dans la ville "par la même rue qui sept mois auparavant } \\
\text { avait vu passer l'empereur Napoléon allant de Cannes à Paris». }\end{array}$ \\
\hline $\begin{array}{l}\text { En entrant dans la ville, le } \\
\text { voyageur avait-il fait des } \\
\text { haltes? }\end{array}$ & Oui, le voyageur avait fait deux haltes. \\
\hline $\begin{array}{l}\text { A quels endroits le voyageur } \\
\text { s'était-il arrêté et pourquoi? }\end{array}$ & $\begin{array}{l}\text { Il s'était arrêté « sous les arbres du boulevard Gassendi » et « cent pas } \\
\text { plus loin (...) à la place du marché», pour boire à la fontaine. }\end{array}$ \\
\hline $\begin{array}{l}\text { Qu'avait-il dégusté au } \\
\text { restaurant de la ville? }\end{array}$ & Le voyageur n'était entré dans aucun restaurant de la ville. \\
\hline $\begin{array}{l}\text { Quelle était la profession du } \\
\text { voyageur? }\end{array}$ & L'auteur ne précise pas la profession du voyageur. \\
\hline Pourquoi voyageait-il ? & L'auteur n'a pas évoqué le but de son voyage. \\
\hline
\end{tabular}




\begin{tabular}{|l|l|}
\hline $\begin{array}{l}\text { À quel moment de la journée } \\
\text { était-il entré dans la ville? }\end{array}$ & $\begin{array}{l}\text { Il était entré dans la ville « une heure environ avant le coucher du soleil } \\
\text { ». }\end{array}$ \\
\hline $\begin{array}{l}\text { Comment s'appelle cette ville } \\
?\end{array}$ & Cette ville s'appelle Digne. \\
\hline $\begin{array}{l}\text { Comment la ville est-elle } \\
\text { décrite? }\end{array}$ & $\begin{array}{l}\text { C'est une " petite ville », ensoleillée, avec un ancien bourg, des } \\
\text { maisons avec des fenêtres donnant sur les rues, un boulevard } \\
\text { (Gassendi), des fontaines et un marché avec une place. }\end{array}$ \\
\hline $\begin{array}{l}\text { Quel passage montre que ce } \\
\text { voyageur venait de loin? }\end{array}$ & $\begin{array}{l}\text { Ce passage montre que ce voyageur venait de loin : « cet homme } \\
\text { avait dû marcher tout le jour. Il paraissait très fatigué ». }\end{array}$ \\
\hline $\begin{array}{l}\text { Etait-ce un jeune homme ou } \\
\text { un vieillard? }\end{array}$ & $\begin{array}{l}\text { Il était « dans la force de l'âge » : « il pouvait avoir quarante-six ou } \\
\text { quarante- huit ans ». }\end{array}$ \\
\hline $\begin{array}{l}\text { Le voyageur était-il grand ou } \\
\text { petit? }\end{array}$ & il était de «moyenne taille, trapu et robuste ». \\
\hline $\begin{array}{l}\text { Le voyageur était-il riche ou } \\
\text { pauvre? }\end{array}$ & Le voyageur était pauvre, car son \\
\hline $\begin{array}{l}\text { Le voyageur était-il d'allure } \\
\text { avenante? }\end{array}$ & $\begin{array}{l}\text { Il n'était pas d'allure avenante parce que les " habitants (...) } \\
\text { regardaient ce voyageur avec une sorte d'inquiétude ». }\end{array}$ \\
\hline
\end{tabular}

\section{Annexe $n^{\circ} 4$}

\begin{tabular}{|c|c|c|}
\hline DESCRIPTION PHYSIQUE & \begin{tabular}{|l} 
TENUE \\
VESTIMENTAIRE
\end{tabular} & CONDITION SOCIALE \\
\hline 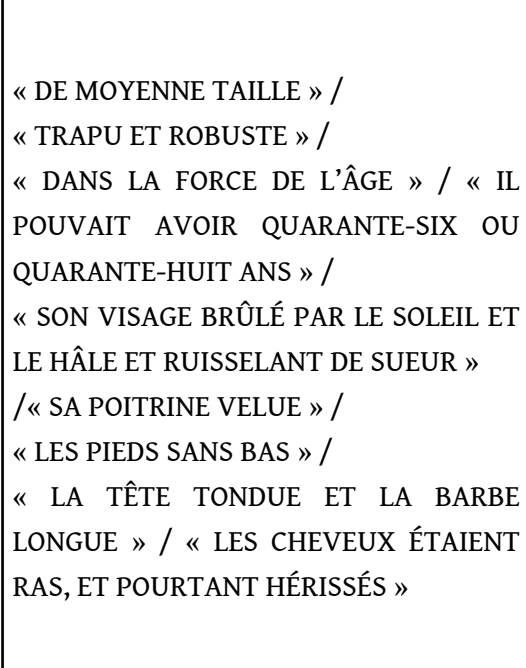 & $\begin{array}{l}\text { « SA CHEMISE DE } \\
\text { GROSSE TOILE JAUNE » } \\
\text { « UNE CRAVATE } \\
\text { TORDUE EN CORDE » } \\
\text { « UN PANTALON DE } \\
\text { COUTIL BLEU» } \\
\text { « UNE VIEILLE BLOUSE } \\
\text { GRISE » }\end{array}$ & $\begin{array}{l}\text { « VOYAGE À PIED » } \\
\text { « PASSANT D’UN ASPECT (...) } \\
\text { MISÉRABLE » / / IL PARAISSAIT } \\
\text { TRÈS } \\
\text { FATIGUÉ »/ " ENSEMBLE } \\
\text { DÉLABRÉ », «SORDIDE » / } \\
\text { « USÉ ET RÂPÉ, BLANC À UN } \\
\text { GENOU, TROUÉ À } \\
\text { L'AUTRE »/ } \\
\text { « EN HAILLONS, RAPIÉCÉE À } \\
\text { L'UN DES COUDES D’UN } \\
\text { MORCEAU DE DRAP VERT } \\
\text { COUSU AVEC DE LA FICELLE » }\end{array}$ \\
\hline
\end{tabular}




\section{Annexe $n^{\circ} 5$}

\begin{tabular}{|c|c|}
\hline $\begin{array}{l}\text { Epithète : « un grand lion est dans la } \\
\text { fosse » }\end{array}$ & $\begin{array}{l}\text { Attribut: « le lion est } \\
\text { malade depuis deux jours » }\end{array}$ \\
\hline \multicolumn{2}{|l|}{ les premiers jours } \\
\hline la petite ville & Il était difficile \\
\hline Les rares habitants & $\begin{array}{l}\text { Les cheveux étaient ras, et pourtant hérissés (...) et } \\
\text { semblaient n'avoir pas été coupés }\end{array}$ \\
\hline $\begin{array}{l}\text { un homme de moyenne taille, trapu, } \\
\text { robuste }\end{array}$ & Il paraissait très fatigué \\
\hline \multicolumn{2}{|l|}{ un aspect (plus) misérable } \\
\hline \multicolumn{2}{|l|}{ Une casquette à visière de cuir rabattue } \\
\hline \multicolumn{2}{|l|}{ visage brûlé (...) ruisselant de sueur } \\
\hline \multicolumn{2}{|l|}{$\begin{array}{l}\text { Sa chemise de toile jaune rattachée au } \\
\text { col }\end{array}$} \\
\hline \multicolumn{2}{|l|}{ sa poitrine velue } \\
\hline \multicolumn{2}{|l|}{$\begin{array}{l}\text { un pantalon bleu usé et râpé, blanc, } \\
\text { troué }\end{array}$} \\
\hline \multicolumn{2}{|l|}{$\begin{array}{l}\text { une vieille blouse grise rapiécée à l'un } \\
\text { des coudes }\end{array}$} \\
\hline \multicolumn{2}{|l|}{ morceau de drap vert cousu } \\
\hline \multicolumn{2}{|l|}{$\begin{array}{l}\text { sac de soldat fort plein, bien bouclé et } \\
\text { tout neuf }\end{array}$} \\
\hline \multicolumn{2}{|l|}{ un énorme bâton noueux } \\
\hline \multicolumn{2}{|l|}{ des souliers ferrés } \\
\hline \multicolumn{2}{|l|}{ la tête tondue } \\
\hline \multicolumn{2}{|l|}{ la barbe longue } \\
\hline \multicolumn{2}{|l|}{$\begin{array}{l}\text { ajoutaient je ne sais quoi de sordide à } \\
\text { cet ensemble délabré }\end{array}$} \\
\hline \multicolumn{2}{|l|}{ quelque temps } \\
\hline l'ancien bourg & \\
\hline
\end{tabular}


Adjectif apposé : « malade, le lion n'a pas quitté la fosse » : cette position n'est pas représentée dans le texte.

\section{Annexe $n^{\circ} 6$}

\begin{tabular}{|c|c|c|}
\hline DIALOGUES & TEXTE & OBSERVATIONS \\
\hline $\begin{array}{l}\text { "J'AI VU UN } \\
\text { VOYAGEUR } \\
\text { DANS CETTE } \\
\text { RUE», } \\
\text { «AVANT LE } \\
\text { COUCHER DU } \\
\text { SOLEIL ». }\end{array}$ & $\begin{array}{l}\text { DANS LES PREMIERS JOURS DU } \\
\text { MOIS D'OCTOBRE 1815, UNE } \\
\text { HEURE ENVIRON AVANT LE } \\
\text { COUCHER DU SOLEIL, UN } \\
\text { HOMME QUI VOYAGEAIT À PIED } \\
\text { ENTRAIT DANS LA PETITE VILLE } \\
\text { DE DIGNE. }\end{array}$ & $\begin{array}{l}\text { LE NARRATEUR SE PRÉOCCUPE DE CAMPER LE } \\
\text { CADRE SPATIO-TEMPOREL DE LA FAÇON LA PLUS } \\
\text { PRÉCISE ET LA PLUS VRAISEMBLABLE, EN } \\
\text { DONNANT DES DÉTAILS TEMPORELS : L'ANNÉE, } \\
\text { LE MOIS ET LE MOMENT. CE DONT LE DIALOGUE } \\
\text { SE SOUCIE PEU PARCE QU'IL A UN AUTRE } \\
\text { CENTRE D'INTÉRÊT : LE VOYAGEUR. }\end{array}$ \\
\hline $\begin{array}{l}\text { "J'ÉTAIS À MA } \\
\text { FENÊTRE ET JE } \\
\text { L'AI APERÇU DE } \\
\text { LOIN ». } \\
\text { «J'ÉTAIS } \\
\text { DESCENDU AU } \\
\text { SEUIL DE MA } \\
\text { MAISON QUAND } \\
\text { IL } \\
\text { S'ÉTAIT } \\
\text { APPROCHÉ » }\end{array}$ & $\begin{array}{l}\text { LES RARES HABITANTS QUI SE } \\
\text { TROUVAIENT EN CE MOMENT À } \\
\text { LEURS FENÊTRES OU SUR LE } \\
\text { SEUIL DE LEURS MAISONS } \\
\text { REGARDAIENT } \\
\text { CE VOYAGEUR AVEC UNE SORTE } \\
\text { D'INQUIÉTUDE. }\end{array}$ & $\begin{array}{l}\text { LE NARRATEUR VA ADOPTER LE POINT DE VUE } \\
\text { DES HABITANTS POUR } \\
\text { DRAMATISER LA DESCRIPTION DE L'INCONNU } \\
\text { PAR LE SENTIMENT QU'IL LEUR INSPIRE ET QUI } \\
\text { INTRODUIT LE SUSPENS DANS LA NARRATION DE } \\
\text { SON } \\
\text { ARRIVÉE À DIGNE. DANS LE } \\
\text { DIALOGUE, LE LOCUTEUR A NE FAIT QUE } \\
\text { PRÉCISER SA POSITION POUR JUSTIFIER LE } \\
\text { DÉTAIL DE SA DESCRIPTION } \\
\text { DU VOYAGEUR. }\end{array}$ \\
\hline $\begin{array}{l}\text { «IL AVAIT UN } \\
\text { ASPECT } \\
\text { MISÉRABLE } \\
\text { ». }\end{array}$ & $\begin{array}{l}\text { IL ÉTAIT DIFFICILE DE } \\
\text { RENCONTRER UN PASSANT D' } \\
\text { UN ASPECT PLUS MISÉRABLE. }\end{array}$ & $\begin{array}{l}\text { LE NARRATEUR DRAMATISE ÉGALEMENT } \\
\text { L'ASPECT DU VOYAGEUR EN } \\
\text { L'INTENSIFIANT, TANDIS QUE LE DIALOGUE } \\
\text { DÉCRIT POUR INFORMER. }\end{array}$ \\
\hline
\end{tabular}




\begin{tabular}{|c|c|c|}
\hline $\begin{array}{l}\text { «SA } \\
\text { CHEMISE ÉTAIT } \\
\text { RATTACHÉE AU } \\
\text { COL PAR UNE } \\
\text { ANCRE } \\
\text { D'ARGENT } \\
\text { ». } \\
\text { «IL } \\
\text { PORTAIT UNE } \\
\text { CHEMISE DE } \\
\text { GROSSE TOILE } \\
\text { JAUNE ». } \\
\text { «IL } \\
\text { PORTAIT UN } \\
\text { PANTALON } \\
\text { BLEU USÉ ». } \\
\text { «SA } \\
\text { BLOUSE AUSSI } \\
\text { ÉTAIT VIEILLE } \\
\text { ET } \\
\text { RAPIÉCÉE ». }\end{array}$ & $\begin{array}{l}\text { SA CHEMISE DE GROSSE TOILE } \\
\text { JAUNE, RATTACHÉE AU COL PAR } \\
\text { UNE PETITE ANCRE D'ARGENT, } \\
\text { LAISSAIT VOIR SA POITRINE } \\
\text { VELUE } \\
; \text { IL AVAIT UNE CRAVATE } \\
\text { TORDUE } \\
\text { EN CORDE, UN PANTALON DE } \\
\text { COUTIL BLEU USÉ ET RÂPÉ, } \\
\text { BLANC } \\
\text { À UN GENOU, TROUÉ À L' } \\
\text { AUTRE, UNE VIEILLE BLOUSE } \\
\text { GRISE EN HAILLONS, RAPIÉCÉE } \\
\text { À L'UN DES COUDES D'UN } \\
\text { MORCEAU DE DRAP } \\
\text { VERT COUSU AVEC DE LA } \\
\text { FICELLE, } \\
\text { SUR LE DOS UN SAC DE SOLDAT } \\
\text { FORT PLEIN, BIEN BOUCLÉ ET } \\
\text { TOUT NEUF, À LA MAIN UN } \\
\text { ÉNORME BÂTON NOUEUX, LES } \\
\text { PIEDS SANS BAS DANS DES } \\
\text { SOULIERS FERRÉS, LA TÊTE } \\
\text { TONDUE ET LA BARBE LONGUE. }\end{array}$ & $\begin{array}{l}\text { LA PRÉCISION DE LA DESCRIPTION DU } \\
\text { PERSONNAGE FAIT ÉCHO À CELLE DE L'ESPACE- } \\
\text { TEMPS, AU NIVEAU } \\
\text { VESTIMENTAIRE (MATIÈRES, COULEURS ET ÉTAT } \\
\text { DES VÊTEMENTS) ET AU NIVEAU PHYSIQUE. } \\
\text { LA CONCISION DES DÉTAILS } \\
\text { FACILITE LA } \\
\text { REPRÉSENTATION QUASI PICTURALE DU } \\
\text { VOYAGEUR QUI NE LAISSE PAS } \\
\text { INDIFFÉRENTS LES HABITANTS DE DIGNE ET LE } \\
\text { LECTEUR. PAR } \\
\text { COMPARAISON, NOUS POUVONS RELEVER } \\
\text { L'INDIGENCE DE LA } \\
\text { DESCRIPTION DU VOYAGEUR DANS LE DIALOGUE } \\
\text { QUI SE CONTENTE DE SON ASPECT } \\
\text { GÉNÉRAL. }\end{array}$ \\
\hline
\end{tabular}

\section{NOTES}

1. Les didacticiens, comme Christian Puren et Janine Courtillon, conçoivent l'« unité didactique » comme unité cohérente d'enseignement déterminée par des objectifs et structurée selon un parcours jusqu'à l'obtention des résultats.

2. Nous avons déterminé le niveau avancé en fonction de la capacité de l'apprenant à distinguer, même de manière sommaire, les différents registres de la langue française, la langue usuelle de la communication et la langue littéraire.

3. Ces dialogues peuvent être considérés aussi comme des modifications du texte prônées par la méthodologie active pour familiariser, au préalable, l'apprenant aux éléments qui l'aideront à comprendre le texte.

4. «Explication de textes et perspective actionnelle : la littérature entre le dire scolaire et le faire social », mis en ligne par l'auteur le 8 octobre 2006. Consultable sur le site : < http://portail-dufle.info/glossaire/purentexteactionnel2006.pdf >

5. «Toute l'approche communicative, en effet, souligne C. Puren, s'était efforcée de neutraliser cette distinction entre l'agir d'apprentissage (ou agir scolaire) et l'agir d'usage (ou social), son activité de référence, la simulation, consistant pour l'élève à parler en tant qu'apprenant comme s'il était un usager, à y réaliser en classe des actes de parole comme s'il agissait ainsi en société »: " Explication de texte et perspective actionnelle ", op.cit.

6. Au chapitre 2, le Cadre Européen Commun de Recherche $(1976,2001)$ justifie son choix de la perspective actionnelle en ces termes : «La perspective privilégiée ici est, très généralement aussi, de type actionnel en ce qu'elle considère avant tout l'usager et l'apprenant d'une langue comme des acteurs sociaux ayant à accomplir des tâches (qui ne sont pas seulement langagières) dans des circonstances et un environnement donné à l'intérieur d'un domaine d'action particulier » (p. 15). 
7. Dans La Didactique des langues étrangères à la croisée des méthodes. Essai sur l'éclectisme (1994), C. Puren fait le point sur la "méthode éclectique ».

8. Selon C. Tagliante, ce type de lecture fait partie de « la compétence de lecture » d'un texte en langue étrangère qui en compte quatre autres : «la lecture écrémage » (aller à l'essentiel), « la lecture survol » (comprendre les idées maîtresses), "la lecture approfondissement » (analyse détaillée, mémorisation) et « la lecture de loisir et de détente » (2001:126-127).

9. Nous nous référons à la typologie de Jean-Michel Adam qui distingue cinq types de textes : narratif, descriptif, explicatif, argumentatif et dialogal (1992:30).

\section{RÉSUMÉS}

Le présent article est une esquisse d'analyse stylistique d'un extrait des Misérables de Victor Hugo à des fins didactiques, en Français Langue Étrangère (FLE). Notre objectif est de démontrer que le principe méthodologique, qui consiste à lier l'étude de la langue à l'étude de la littérature, favorise l'acquisition de compétences aussi bien orales qu'écrites. A cet effet, nous avons choisi l'enseignement/apprentissage implicite de la catégorie grammaticale des adjectifs et la syntaxe littéraire à travers un texte littéraire descriptif de Victor Hugo (le portrait de Jean Valjean à son arrivée à Digne). Notre méthode est une combinaison de différentes méthodologies: la méthodologie active, l'approche communicative et la perspective actionnelle.

The present paper is a stylistic analysis of an extract of Victor Hugo's Les Misérables for educational purposes in teaching French as a foreign language (F.F.L). Our goal is to demonstrate that the methodological principle of linking the study of language with the study of literature, promotes the acquisition of both oral and written skills. For this purpose, we opted for the teaching/learning of adjectives as a grammatical category, a descriptive literary text (le portrait de Jean Valjean à son arrivée à Digne), and a method based on various methodologies: active methodology, communicative approach and task-based approach.

\section{INDEX}

Keywords : FFL, stylistic landmark, didactics, Victor Hugo, Les Misérables

Mots-clés : littérature française, stylistique, didactique, méthodologies d'enseignement, Victor Hugo, Les Misérables

\section{AUTEUR}

\section{AYIH AYITEY}

Université du Ghana - Département de français Ghana 